\title{
Anthropogenic contamination of groundwater with nitrate in arid region: case study of southern Hodna (Algeria)
}

\author{
Salah Abdesselam • Amor Halitim • \\ Anthony Jan · Fabienne Trolard · Guilhem Bourrié
}

Received: 7 April 2011/Accepted: 7 July 2012/Published online: 25 July 2012

(C) The Author(s) 2012. This article is published with open access at Springerlink.com

\begin{abstract}
In southern Hodna, an arid region of Algeria, the extension of irrigated agriculture and overexploitation of groundwaters have led to a rupture with traditional land use by pastoralism. Due to the arid conditions, groundwaters are the only water resources for man and irrigation. This study focuses on nitrate contamination of groundwaters and its increase since the last 40 years, on the basis of two archives and recent analyses. The chemical facies of waters is sulphate-chloride, the risk of salinity is moderate to large. The sodicity risk, as estimated by the SAR, is low due to the large salinity and the sandy soil texture. Nitrate contamination of deep groundwater is a clear evidence when recent data are compared to the historical data. From 1996 to 2008, the proportion of samples with $\mathrm{NO}_{3}^{-}$concentrations larger than $50 \mathrm{mg} / \mathrm{L}$ increases from 24 to $61 \%$, the proportion of samples with $\mathrm{NO}_{3}^{-}$concentrations larger than $100 \mathrm{mg} / \mathrm{L}$ increases from 12 to $27 \%$. The study points at a general contamination of aquifers from anthropic origin: phreatic water was already contaminated when pastoralism was dominant (1967); as population increased and irrigation agriculture developed with large use of $\mathrm{N}$-fertilizers and organic amendments, contamination extended spatially and vertically to deep aquifer. To remediate this contamination, fertilizers should be fractionated and limited to plant requirements on the basis of soil and plant
\end{abstract}

S. Abdesselam - A. Halitim

Batna University, Batna, Algeria

e-mail: aksalah2001@yahoo.fr

A. Jan · F. Trolard · G. Bourrié $(\bowtie)$

INRA, UMR 1114 Emmah, Avignon, France

e-mail: bourrie@paca.inra.fr

A. Jan · F. Trolard · G. Bourrié

UAPV, UMR 1114 Emmah, Avignon, France analyses. Excess of irrigation should be avoided. Soil surface should be continuously covered to prevent nitrate leaching. Secondly, more efficient irrigation techniques, such as localized irrigation, should be implemented, with adaptation of crop systems and fertilizer inputs to arid conditions. Farmers should then be trained; simultaneously, local agronomic references that are presently lacking should be acquired.

Keywords Water quality - Groundwater . Contamination $\cdot$ Nitrate $\cdot$ Hodna $\cdot$ Algeria

\section{Introduction}

In rural areas, water management is largely influenced by agriculture, and intensification of agriculture requires a better control of water resources and especially in arid or semi-arid regions of irrigation when possible. However, bad irrigation management and excess of fertilizers result in degradation of water quality, mostly by contamination by nitrate. Moreover, in arid or semi-arid conditions, small pluviometry and large evapotranspiration favour this degradation. Consequently, irrigation often results in raise of the water table when drainage is poor and an increase of soil salinity (Daoud and Halitim 1994; Djili et al. 2003) and contamination of groundwater (Rouabhia et al. 2004; Baali et al. 2007).

In Southern Hodna, where irrigated agriculture has developed since some 40 years, two aquifers are present, the phreatic aquifer and the deep aquifer. The first one shows a large salt content. It has strongly decreased due to regional lowering by pumping (ANRH 2006), so it is presently limited to the immediate proximity of Chott El Hodna. The deeper one shows a small salt content. At 
present it is the only resource to meet man and crops needs. Its renewal is easy due to the large extension of the watershed. It has progressively lowered due to overexploitation by pumping from an increasing number of boreholes (ANRH 2007). The largest lowering of the deep aquifer is observed in the cultivated area, where intensive agriculture has developed to benefit from this water resource and from the increase of the population. Concomitantly, contamination of water by nitrate is increasingly observed (Abdesselam et al. 2008). This study aims at a better diagnosis of this contamination and of its causes.

\section{Study area}

Physiography

Hodna region, in the arid zone of Algeria, is a marked depression of $c a .8,500 \mathrm{~km}^{2}$ surrounded by mountains. In its centre is situated the Chott El Hodna, of $c a .1,100 \mathrm{~km}^{2}$. It is the outlet of many temporary rivers ("Oueds"), the largest of which come from the North. The main districts of Southern Hodna are Mâarif, Khoubana, M'cif, Houamed and Boussâada (Fig. 1).

The region South of Hodna, called R'Mel ("sand") region, consists of sand dunes, recent alluvial deposits and isolated rocky hills (e.g. Djbel Meharga, $900 \mathrm{~m}$ ). To the south, at ca. 550-850 m, the landscape consists of rocky hills and large glacis (or pediment) (Grandin and Joly 2008) made of sand, gravels, pebbles locally cemented. This area is permanently submitted to wind erosion, due to the sandy texture, the absence of vegetation and soil tillage, and to sedimentation (sheet wash and wind). It is drained by numerous oueds that leach salts at depth and bring silts to soils.

\section{Climate}

The climate of Southern Hodna is subdesertic arid. Its main characteristic is the extreme variability of rainfall both in space and time (Sebhi 1987). Average pluviometry is $c a$. $172 \mathrm{~mm}$, average annual temperature $19.4{ }^{\circ} \mathrm{C}$ and average potential evapotranspiration 1,330 $\mathrm{mm} /$ year (Abdesselam et al. 2007).

Soils

Soil texture is dominated by sand. Apart from quartz, soil components are dominated by carbonates (calcite), gypsum and salts. Due to the sandy texture and the small organic matter content, soil fertility is low with small CEC and water holding capacity; inputs of fertilizers and organic amendments are thus necessary to obtain correct yields, but fertilization is empirical.

According to soil study of Hodna (Boyadgiev 1975), and to WRB (2006), the main soil types present in the irrigated area are:

- Haplic Arenosols (Aridic):

- Haplic Fluvisols (Aridic, Gypsic);

- Haplic Gypsisols (Aridic);

- Gypsic Gleysols (Aridic);

- Gypsic Solonchaks (Aridic);

- Haplic Solonchaks (Aridic).

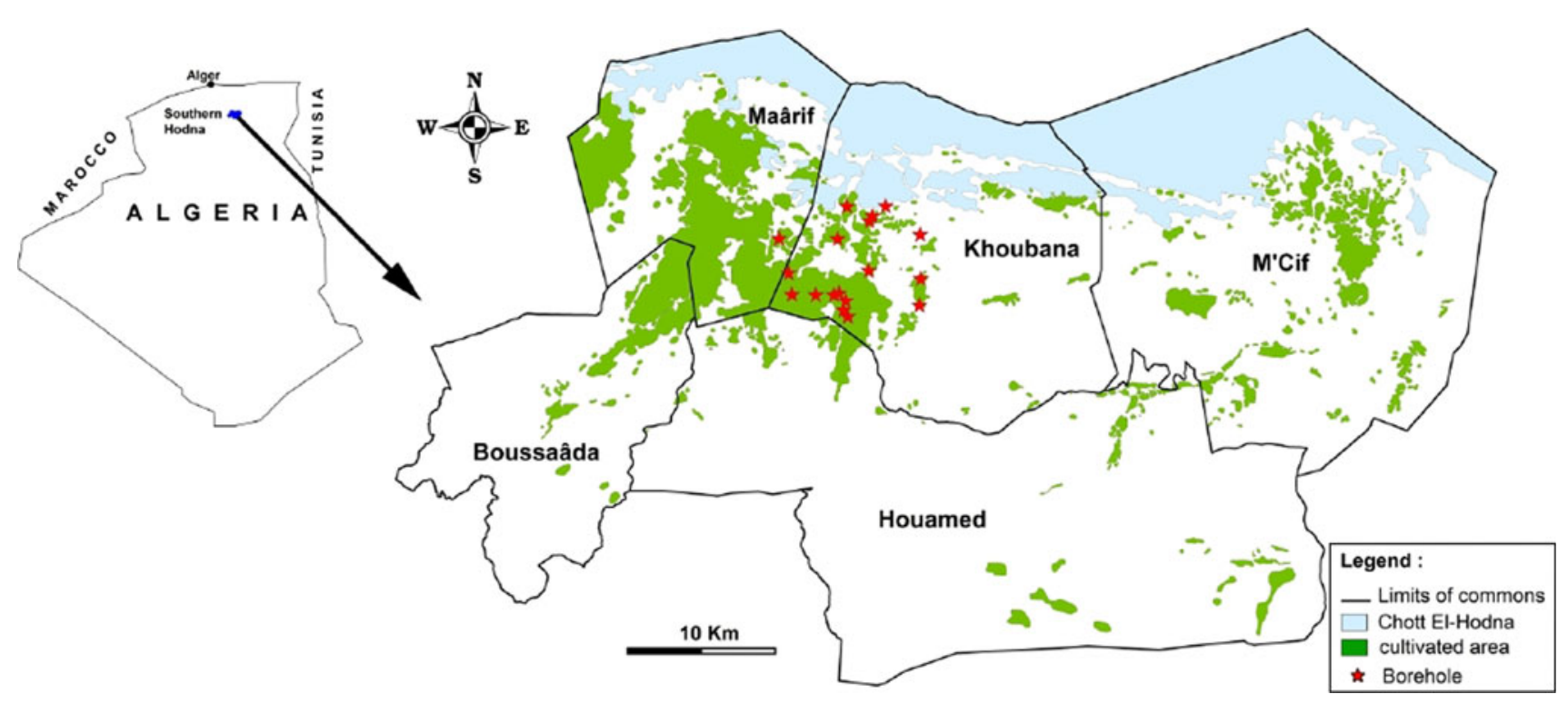

Fig. 1 Situation of the study area, south of Chott El Hodna. Boreholes sampled in 2008 are indicated by red stars 


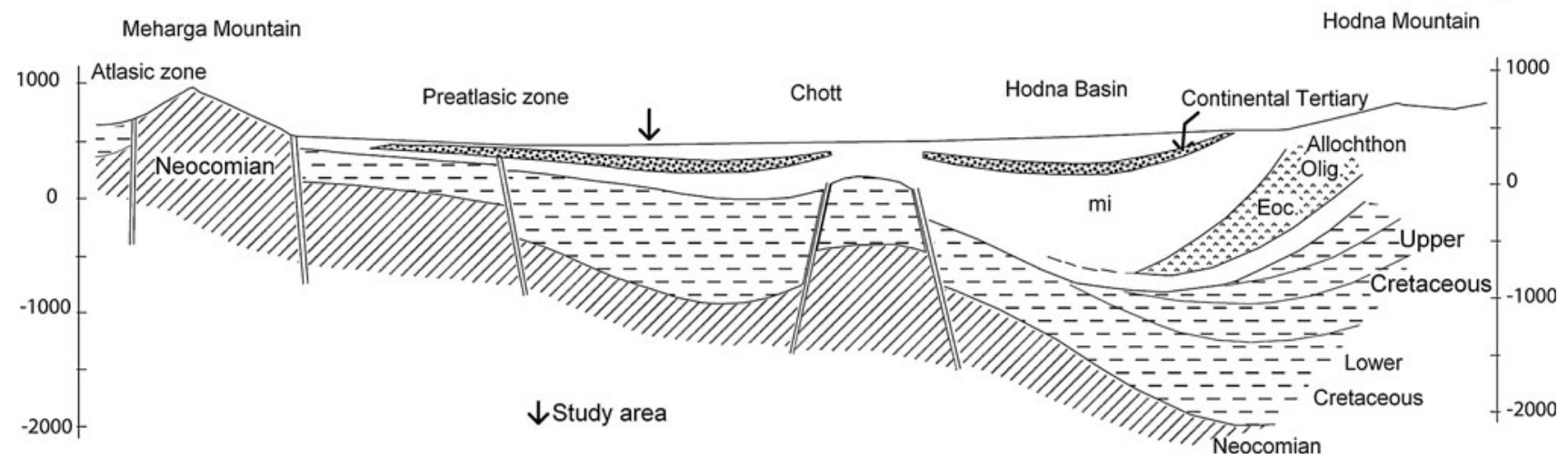

Fig. 2 Schematic cross section of the Chott El Hodna Basin. Elevation and depth, /metre

\section{Water resources}

The first evaluation of water resources (FAO 1972, 1973) aimed at serving as a basis for the agricultural development of the region. In the South Hodna, the aquifer consists of Quaternary sands and sandy clays of small thickness. The phreatic groundwater is very shallow with a high salinity: TDS ranges from 0.5 to $250 \mathrm{~g} / \mathrm{L}$. The deep aquifer consists of intercalated marls and conglomerates (Continental Tertiary) (Fig. 2). This formation, ca. 100-400 m thick, lies upon nearly all older formations. It collects water from them and constitutes the major aquifer. Water is in charge between the southern reliefs and the Chott El Hodna (FAO 1968). It is a renewable aquifer (FAO 1972; ANRH 2006). Its recharge implies three contributions:

- direct infiltration of rainfall;

- infiltration of water from oueds;

- deep recharge by surrounding formations.

Groundwater exploitation led to the extension of irrigated areas and land use change at the expense of pastoralism. Borehole water salinity is between 0.5 and $5 \mathrm{~g} / \mathrm{L}$. Isopiezometric lines of the two groundwaters converge in the Chott (FAO 1973). The Chott is the natural outlet of water evaporation for the phreatic groundwater and for the groundwater in charge. Presently, lowering of the groundwater level larger than $15 \mathrm{~m}$ since the 1970s was registered (ANRH 2006). Simulation of scenarios of water pumping (ANRH 2007) show that lowering of the groundwater level below the level of the Chott could result in a reversal of the flow direction with saline water from the Chott flowing to the aquifer, and serious damage to economic development of the region.

Indeed, the limits between phreatic aquifer and deep aquifer are always unclear (FAO 1970), as they are not separated by a continuous formation of small permeability, but by discontinuous lenses of variable permeability.
Consequently, the two groundwaters may communicate and contamination of the deep aquifer from the surface is possible.

Land use change and agricultural development

This region is subject to heavy pressures, both natural such as aridity of climate, eolian erosion, and anthropic such as overgrazing, overexploitation of water resources, and intensive agriculture (Abdesselam et al. 2007). Since several decades, important changes in land use and exploitation of water resources have occurred, driven by successive agricultural policies in Algeria, the agricultural revolution, access to private land property, and the national program of agricultural development.

It is since $c a$. 1976 that irrigation was initiated, in the framework of agricultural revolution, with the purpose to irrigate 300 ha by exploitation of the underlying artesian groundwater (Sebhi 1987). During the last 30 years, agricultural lands extended at the expense of land used for pastoralism. Sandy formations extended too (Abdesselam et al. 2009). The reduction of pastoral devoted areas is mainly due to the extension of cultivated areas. Moreover soil tillage resulted in increasing eolian erosion (Mimoune 1995) and the formation of sand dunes. The degradation of pastoral areas is not only due to climatic conditions, but mainly to overgrazing.

Agriculture has developed where water resources are accessible, i.e. in the sandy area, south of the Chott El Hodna. From the statistics of the Direction des Services Agricoles (DSA), the arable land area (ALA) is ca. 42,000 ha, of which $50 \%$ is irrigated.

\section{Materials and methods}

Archive data both from wells and boreholes were collected and recently boreholes were sampled. Wells sample mainly 


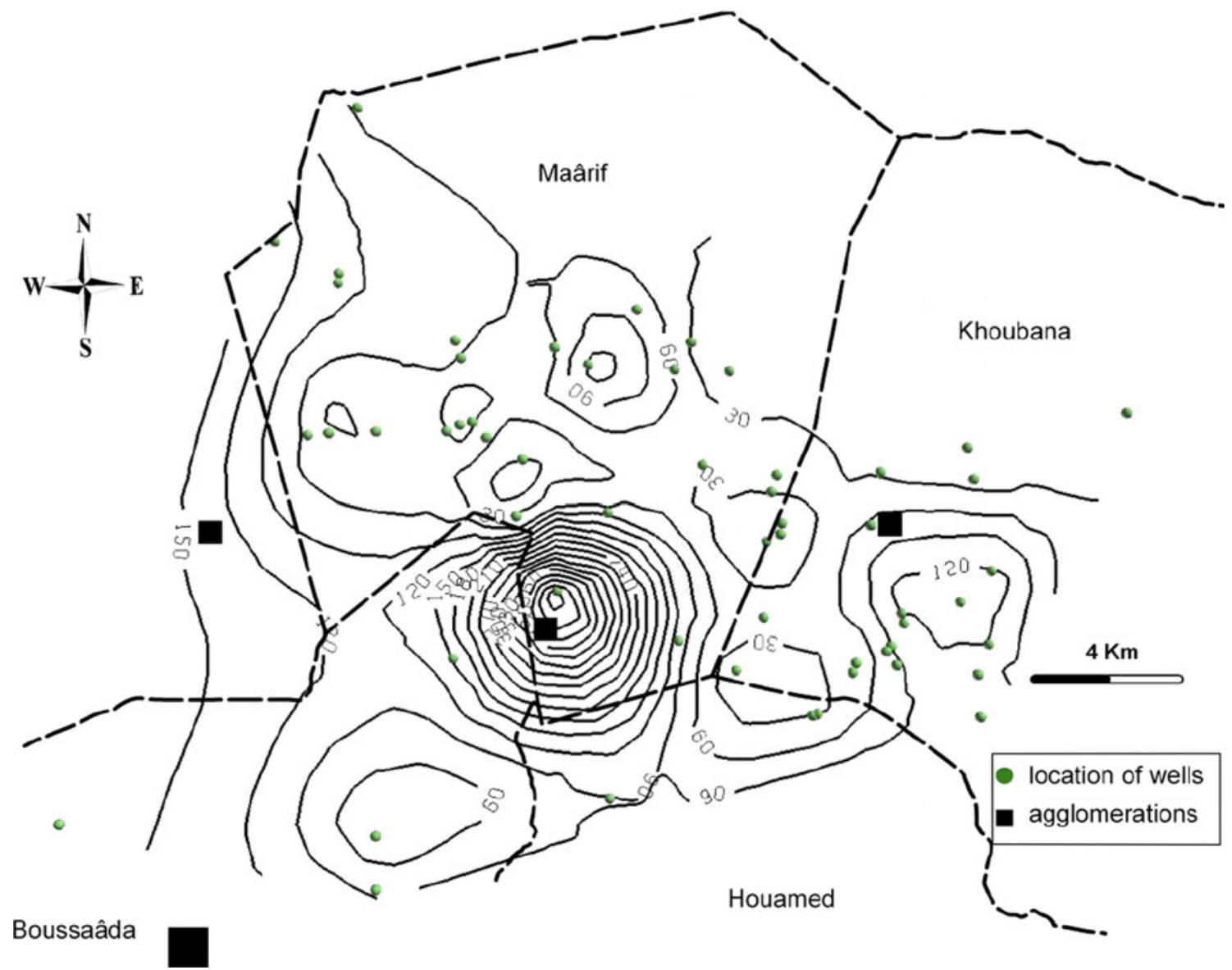

Fig. 3 Situation of wells and spatial variability of nitrate in phreatic groundwater in 1967. Data from FAO (1973), $\mathrm{NO}_{3}^{-}$concentrations $/ \mathrm{mg} \mathrm{L}^{-1}$

the phreatic, shallow aquifer, and boreholes sample mainly the deep aquifer, but those aquifers are not separated by an impervious formation. Contamination of the deep aquifer by the phreatic one is thus possible. The risk of contamination is enhanced by the unavoidable imperfections in the boring techniques or errors.

\section{Archive data}

The archive data consist of (1) analyses of 63 wells (FAO 1973) sampled in the whole western part of the agricultural area (Maârif-Khoubana, Boussaâda and Houamed) (Fig. 3); (2) analyses of 50 boreholes (ANRH 1996), sampled in the extreme west (Maârif) (Fig. 4).

\section{Present data}

Samples were taken in 18 boreholes in the deep groundwater after $1 \mathrm{~h}$ pumping, in polyethylene bottles, in May 2008, near Khoubana, south of Hodna (Fig. 1), from the Chott to Mergueb, Guebouhia and Memdouda. Temperature, $\mathrm{pH}$ and electrical conductivity were measured in the field, with a portable pH-meter/conductimeter HANNA.
Samples were immediately transferred to the laboratory of ANRH (Agence Nationale des Ressources Hydrauliques) for analysis of major ions. $\mathrm{Ca}$ and $\mathrm{Mg}$ were analyzed by complexation and spectrophotometry with a SKALAR SAN continuous flux analyzer; $\mathrm{Na}$ and $\mathrm{K}$ were analyzed by flame photometry, sulphate by turbidimetry, chloride by colorimetry, nitrate by continuous flux analysis at $540 \mathrm{~nm}$ and alkalinity by volumetry. Total dissolved salts (TDS) were measured by gravimetry after evaporation.

Spatial interpolation

Spatial interpolation of data was achieved with geomodel gOcad (Mallet 2002) in order to compare data obtained at different dates and locations.

\section{Results and discussion}

Chemical facies of groundwater

Complete analyses are given in Tables 1,2 and 3. Synthetic statistical parameters are given in Table 4. The 


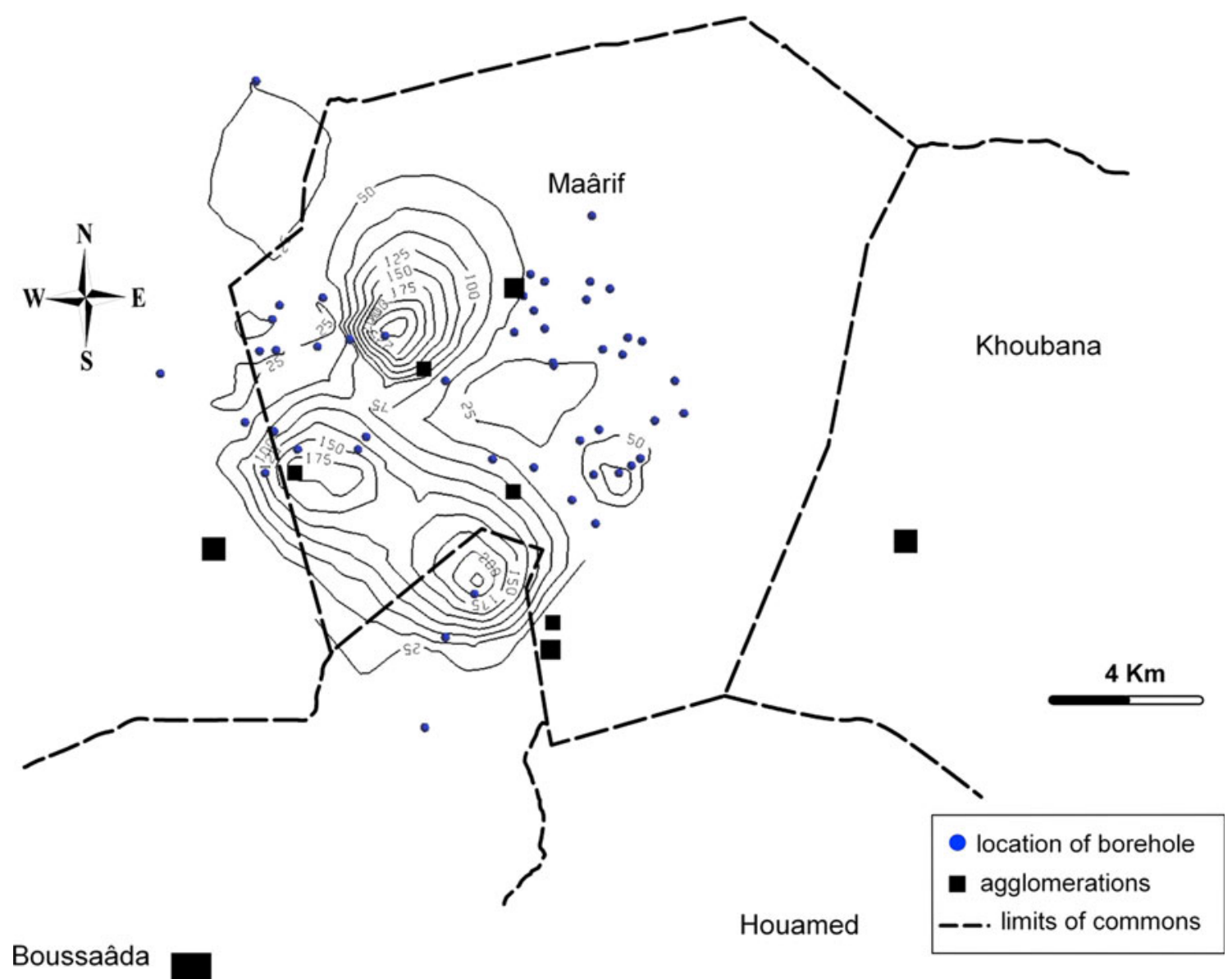

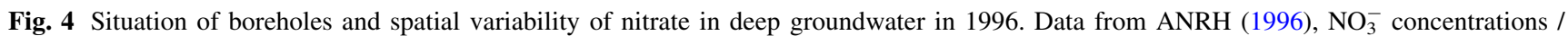
$\mathrm{mg} \mathrm{L}^{-1}$

relative proportions of cations and anions in the Piper's diagram are given in Fig. 5.

The facies of all waters is sulphate-chloride-calcium, $\mathrm{pH}$ is near neutrality (6.8-8) and alkalinity is moderate.

From Piper's diagram, and standard deviations (see CE and TDS, Table 4) it is clear that the scattering of data is the lowest in 2008. This can be ascribed to:

- better techniques in boreholes;

- sampling in areas where aquifers are better separated;

- an improvement of analytical techniques from 1996 to present.

EC ranges from 644 to $2,780 \mu \mathrm{S} / \mathrm{cm}$ in boreholes in 2008 , as compared to $1,500-9,700 \mu \mathrm{S} / \mathrm{cm}$ in 1996 and from 1,011 to $21,900 \mu \mathrm{S} / \mathrm{cm}$ in wells in 1967 .

TDS ranges from 512 to $2,510 \mathrm{mg} / \mathrm{L}$ in 2008 , as compared to $1,066-6,608 \mathrm{mg} / \mathrm{L}$ in 1996 and 738 to $20,300 \mathrm{mg} / \mathrm{L}$ in wells in 1967.

These large variations contrast with the quasi stability of $\mathrm{pH}$. This implies that despite concentration of solution by evaporation and transpiration, carbonate precipitation regulates $\mathrm{pH}$. According to residual alkalinity concept (Breemen and Wielemaker 1974; Al Droubi et al. 1980), solutions evolve in the neutral pathway and not in the alkaline pathway.

The salinity threshold for water use in agriculture is estimated as $3,000 \mu \mathrm{S} / \mathrm{cm}$ at $25^{\circ} \mathrm{C}$ (Ayers and Westcot 1985). In 1996, $42 \%$ of samples showed a larger value as compared to $63 \%$ in well waters in 1967. This threshold severely limits the use of water for irrigation. Values of $\mathrm{Cl}^{-}$concentration larger than $10 \mathrm{mmole} / \mathrm{L}$ are observed in $63 \%$ of samples in 1967. Those excessive values may be responsible for low yields (Mimoune 1995). According to USSL (1954); Durand (1958); Ayers and Westcot (1985), the salinization hazard is light to moderate in 2008 versus moderate to very large in 1996 and 1967.

The risk of degradation of soil structure due to clay dispersion is classically assessed by considering the sodium adsorption ratio (SAR) (USSL 1954) and the ionic strength, or equivalently the EC. In 2008, SAR ranged from 0.57 to 2.8 and these values are acceptable as the EC is larger than $700 \mu \mathrm{S} / \mathrm{cm}$. 
Table 1 Chemical composition of groundwater from wells, 1967 (FAO 1973)

\begin{tabular}{|c|c|c|c|c|c|c|c|c|c|c|c|c|c|c|}
\hline \multirow{2}{*}{$\begin{array}{l}\text { Site } \\
\text { no. }\end{array}$} & \multicolumn{2}{|c|}{ Coordinates* } & \multirow[t]{2}{*}{$t\left({ }^{\circ} \mathrm{C}\right)$} & \multirow{2}{*}{ 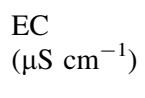 } & \multirow{2}{*}{$\begin{array}{l}\text { TDS } \\
\left(\mathrm{mg} \mathrm{L}^{-1}\right)\end{array}$} & \multirow[t]{2}{*}{$\mathrm{Ca}^{2+}$} & \multirow[t]{2}{*}{$\mathrm{Mg}^{2+}$} & \multirow[t]{2}{*}{$\mathrm{Na}^{+}$} & \multirow[t]{2}{*}{$\mathrm{K}^{+}$} & \multirow[t]{2}{*}{$\mathrm{HCO}_{3}^{-}$} & \multirow[t]{2}{*}{$\mathrm{SO}_{4}^{2-}$} & \multirow[t]{2}{*}{$\mathrm{Cl}^{-}$} & \multirow[t]{2}{*}{$\mathrm{NO}_{3}^{-}$} & \multirow[t]{2}{*}{ SAR } \\
\hline & $X$ & $Y$ & & & & & & & & & & & & \\
\hline 1 & 640625 & 217150 & 20 & 2,180 & 1,157 & 30 & 185 & 143.08 & 0 & 38 & 764 & 210 & 31 & 2.15 \\
\hline 3 & 640625 & 215600 & 20 & 2,530 & 2,040 & 308 & 97 & 177.16 & 0 & 98 & 918 & 275 & 89 & 2.25 \\
\hline 19 & 631650 & 217440 & 21 & & 5,229 & 898 & 124 & 305.6 & 0.24 & 134 & 1,873 & 918 & 177 & 2.53 \\
\hline 22 & 647225 & 218200 & 19 & 3,240 & 2,732 & 362 & 138 & 279.66 & 0.22 & 80 & 1,140 & 426 & 88 & 3.16 \\
\hline 29 & 638700 & 228450 & 20 & 1,445 & 991 & 157 & 51 & 74.6 & 1.37 & 93 & 432 & 89 & 60 & 1.3 \\
\hline 30 & 639575 & 233025 & 17 & 4,090 & 3,189 & 416 & 107 & 285.6 & 0.27 & 93 & 1,361 & 595 & 89 & 2.9 \\
\hline 121 & 641200 & 231500 & 13.5 & 3,080 & 3,084 & 616 & 106 & 34.08 & 0 & 103 & 1,749 & 59 & & 0.33 \\
\hline 122 & 647750 & 223450 & & 3,730 & 3,350 & 386 & 257 & 204.74 & 0.17 & 87 & 1,715 & 266 & & 1.98 \\
\hline 123 & 647400 & 223750 & 15.5 & 5,450 & 5,410 & 508 & 459 & 258.84 & 0.05 & 87 & 2,584 & 463 & & 1.97 \\
\hline 124 & 645750 & 224050 & 19 & 5,050 & 4,261 & 580 & 343 & 209.88 & 0.02 & 81 & 1,899 & 559 & 535 & 1.7 \\
\hline 126 & 649175 & 222660 & 18 & 7,600 & 8,246 & 574 & 635 & 766.28 & 14.42 & 178 & 4,250 & 746 & 100 & 5.22 \\
\hline 127 & 650800 & 221825 & 17 & 4,920 & 4,300 & 370 & 225 & 454.46 & 13.37 & 203 & 1,885 & 381 & 0 & 4.58 \\
\hline 128 & 631575 & 223325 & 18.3 & 4,490 & 3,924 & 417 & 252 & 346.66 & 10.21 & 177 & 1,810 & 375 & 48 & 3.21 \\
\hline 129 & 652075 & 225980 & 18.3 & 6,570 & 6,824 & 648 & 390 & 691.37 & 37.41 & 267 & 2,950 & 884 & 0 & 5.28 \\
\hline 130 & 652050 & 225675 & 18.8 & 4,340 & 4,226 & 519 & 236 & 310.79 & 7.09 & 206 & 1,975 & 325 & 32 & 2.82 \\
\hline 131 & 651650 & 225460 & 16.8 & 4,990 & 5,614 & 648 & 301 & 354.21 & 49.75 & 365 & 2,400 & 582 & 0 & 2.87 \\
\hline 132 & 652950 & 220550 & & 1,920 & 1,337 & 146 & 85 & 166.88 & 0.05 & 124 & 532 & 209 & 44 & 2.6 \\
\hline 133 & 653100 & 220600 & 18 & 1,470 & 867 & 106 & 48 & 120.09 & 0 & 112 & 221 & 220 & 18 & 2.39 \\
\hline 134 & 654200 & 222025 & 18.8 & 1,820 & 1,260 & 133 & 73 & 112.95 & 3.01 & 255 & 390 & 178 & 52 & 1.96 \\
\hline 135 & 654100 & 221760 & 18.8 & 1,180 & 738 & 82 & 56 & 81.04 & 2.93 & 241 & 220 & 99 & 43 & 1.68 \\
\hline 136 & 655040 & 222360 & 18.3 & 2,400 & 1,501 & 217 & 171 & 61.68 & 7.31 & 262 & 580 & 337 & 10 & 0.77 \\
\hline 137 & 655350 & 221960 & 19.2 & 2,250 & 1,450 & 271 & 129 & 70.51 & 2.47 & 238 & 330 & 273 & 104 & 0.98 \\
\hline 138 & 655200 & 222500 & 17.5 & 1,910 & 1,489 & 168 & 137 & 36.21 & 8.8 & 216 & 185 & 220 & 150 & 0.52 \\
\hline 139 & 657960 & 222560 & 19.3 & 1,300 & 943 & 85 & 77 & 70.71 & 12.29 & 213 & 215 & 91 & 132 & 1.34 \\
\hline 140 & 657160 & 223750 & 18.4 & 1,670 & 1,122 & 93 & 73 & 139.65 & 5.3 & 319 & 270 & 169 & 124 & 2.61 \\
\hline 141 & 658040 & 224625 & 18.2 & 3,930 & 3,270 & 228 & 219 & 382.68 & 32.23 & 250 & 1,187 & 568 & 130 & 4.34 \\
\hline 142 & 642875 & 231125 & 17.7 & 6,290 & 5,578 & 529 & 253 & 727.77 & 32.99 & 183 & 2,400 & 866 & 90 & 6.3 \\
\hline 143 & 643025 & 230625 & 17.9 & 5,900 & 5,592 & 545 & 338 & 577.39 & 17.4 & 193 & 2,625 & 650 & 40 & 4.75 \\
\hline 144 & 643350 & 228825 & 18.3 & 2,020 & 1,628 & 222 & 96 & 117.22 & 4.74 & 188 & 700 & 149 & 28 & 1.64 \\
\hline 145 & 643750 & 228375 & 18 & 2,950 & 2,637 & 361 & 139 & 173.17 & 5.77 & 195 & 1,212 & 242 & 45 & 1.69 \\
\hline 146 & 644775 & 227750 & 17 & 7,080 & 7,178 & 556 & 476 & 807.95 & 21.75 & 224 & 3,150 & 1,212 & 0 & 6.06 \\
\hline 147 & 644600 & 226175 & 19.6 & 6,160 & 6,148 & 534 & 398 & 576.97 & 13.81 & 214 & 3,050 & 458 & 0 & 4.72 \\
\hline 148 & 642800 & 222150 & 20.8 & 2,670 & 1,861 & 295 & 105 & 113.12 & 5.84 & 137 & 850 & 219 & 144 & 1.44 \\
\hline 149 & 647175 & 226275 & 20.8 & 3,730 & 3,321 & 361 & 204 & 300.02 & 5.86 & 189 & 1,575 & 249 & 70 & 3.12 \\
\hline 150 & 648800 & 229850 & 16.2 & 5,660 & 5,067 & 500 & 261 & 651.43 & 58.43 & 424 & 2,175 & 824 & & 5.86 \\
\hline 151 & 649060 & 230300 & 18.5 & 5,660 & 4,773 & 578 & 236 & 537.73 & 30.11 & 165 & 2,050 & 909 & 59 & 4.75 \\
\hline 152 & 649525 & 231070 & 15 & 6,160 & 5,710 & 612 & 313 & 599.9 & 38.93 & 244 & 2,950 & 795 & 18 & 4.9 \\
\hline 153 & 648000 & 232000 & 13.5 & 8,850 & 8,596 & 623 & 544 & $1,076.88$ & 73.82 & 184 & 3,375 & 1,447 & 78 & 7.58 \\
\hline 154 & 646625 & 230450 & 14.8 & 17,260 & 13,362 & 756 & 758 & $2,336.52$ & 79.64 & 310 & 3,625 & 4,047 & 152 & 14.25 \\
\hline 155 & 645675 & 230925 & 19.7 & 5,340 & 6,602 & 589 & 390 & 809.18 & 9.48 & 228 & 2,800 & 1,179 & 35 & 6.32 \\
\hline 156 & 645975 & 231775 & 13.3 & 5,660 & 5,068 & 600 & 266 & 514.14 & 78.83 & 319 & 2,550 & 463 & & 4.32 \\
\hline 157 & 651800 & 226850 & 18.7 & 9,130 & 5,048 & 723 & 197 & 445.43 & 63.53 & 145 & 1,925 & 994 & 39 & 3.78 \\
\hline 158 & 651950 & 227325 & 16 & 10,110 & 8,367 & 556 & 458 & $1,180.98$ & 74.68 & 171 & 3,400 & 1,349 & 52 & 9.28 \\
\hline 159 & 649850 & 227600 & 15 & 8,330 & 9,015 & 645 & 595 & $1,460.02$ & 61.48 & 232 & 3,525 & 2,095 & 30 & 9.93 \\
\hline 160 & 661800 & 229075 & 15.9 & 3,960 & 7,796 & 589 & 450 & 980.44 & 78.32 & 232 & 3,200 & 1,357 & 0 & 7.4 \\
\hline 161 & 640625 & 228550 & 18.3 & 1,670 & 1,277 & 268 & 54 & 24.17 & 12.85 & 211 & 600 & 57 & 40 & 0.35 \\
\hline 162 & 654875 & 227400 & 13.5 & 10,110 & 9,151 & 731 & 507 & $1,427.61$ & 75.95 & 250 & 3,750 & 2,024 & 22 & 9.94 \\
\hline
\end{tabular}


Table 1 continued

\begin{tabular}{|c|c|c|c|c|c|c|c|c|c|c|c|c|c|c|}
\hline \multirow{2}{*}{$\begin{array}{l}\text { Site } \\
\text { no. }\end{array}$} & \multicolumn{2}{|c|}{ Coordinates* } & \multirow[t]{2}{*}{$t\left({ }^{\circ} \mathrm{C}\right)$} & \multirow{2}{*}{$\begin{array}{l}\mathrm{EC} \\
\left(\mu \mathrm{S} \mathrm{cm}^{-1}\right)\end{array}$} & \multirow{2}{*}{$\begin{array}{l}\text { TDS } \\
\left(\mathrm{mg} \mathrm{L}^{-1}\right)\end{array}$} & \multirow[t]{2}{*}{$\mathrm{Ca}^{2+}$} & \multirow[t]{2}{*}{$\mathrm{Mg}^{2+}$} & \multirow[t]{2}{*}{$\mathrm{Na}^{+}$} & \multirow[t]{2}{*}{$\mathrm{K}^{+}$} & \multirow[t]{2}{*}{$\mathrm{HCO}_{3}^{-}$} & \multirow[t]{2}{*}{$\mathrm{SO}_{4}^{2-}$} & \multirow[t]{2}{*}{$\mathrm{Cl}^{-}$} & \multirow[t]{2}{*}{$\mathrm{NO}_{3}^{-}$} & \multirow[t]{2}{*}{ SAR } \\
\hline & $X$ & $Y$ & & & & & & & & & & & & \\
\hline 163 & 657500 & 227200 & 16.3 & 9,440 & 8,201 & 567 & 368 & $1,406.00$ & 98.63 & 279 & 3,625 & 1,136 & 12 & 11.72 \\
\hline 164 & 657325 & 228100 & 15 & 10,340 & 9,988 & 523 & 621 & $1,474.91$ & 84.65 & 243 & 3,775 & 1,690 & 20 & 10.63 \\
\hline 165 & 654600 & 225925 & 16 & 8,250 & 8,744 & 567 & 578 & $1,050.76$ & 94.01 & 262 & 3,400 & 1,349 & 66 & 7.4 \\
\hline 166 & 655550 & 223150 & 17.6 & 1,880 & 1,394 & 136 & 83 & 113.22 & 49.85 & 195 & 430 & 136 & 92 & 1.88 \\
\hline 167 & 655500 & 223425 & 17.5 & 2,400 & 1,858 & 167 & 120 & 188.14 & 49.9 & 250 & 625 & 220 & 140 & 2.7 \\
\hline 168 & 657650 & 221700 & 18.4 & 1,880 & 1,426 & 115 & 147 & 46.78 & 0.20 & 290 & 420 & 138 & 92 & 0.68 \\
\hline 169 & 657725 & 220500 & 16.5 & 1,410 & 1,026 & 106 & 70 & 73.65 & 5.33 & 198 & 250 & 106 & 106 & 1.37 \\
\hline 170 & 639300 & 228500 & 16 & 1,970 & 1,606 & 232 & 98 & 80.02 & 8.97 & 189 & 750 & 82 & 18 & 1.17 \\
\hline 172 & 642625 & 228550 & 19.5 & 2,620 & 2,278 & 306 & 152 & 153.79 & 3.15 & 171 & 1,212 & 116 & 23 & 1.79 \\
\hline 173 & 643000 & 228750 & 18.8 & 3,630 & 3,180 & 378 & 201 & 253.12 & 16.81 & 166 & 1,475 & 334 & 124 & 2.59 \\
\hline 174 & 639550 & 232750 & 18.6 & 7,870 & 6,942 & 411 & 638 & 600.25 & 5.5 & 234 & 2,000 & 1,882 & 104 & 4.31 \\
\hline 175 & 637750 & 233950 & 13.2 & 7,410 & 6,228 & 577 & 277 & 784.09 & 46.67 & 222 & 1,810 & 1,385 & 144 & 6.4 \\
\hline 179 & 640100 & 237700 & 12.8 & 8,700 & 8,212 & 580 & 498 & $1,124.46$ & 51.17 & 258 & 3,800 & 1,484 & 50 & 8.25 \\
\hline 185 & 638250 & 230500 & & 3,300 & 2,824 & 314 & 206 & 259.64 & 0.24 & 191 & 1,586 & 426 & & 2.8 \\
\hline 186 & 646350 & 230400 & & 21,900 & 20,300 & 560 & 1,340 & $3,456.51$ & 1.95 & 351 & 7,488 & 4,473 & & 18.03 \\
\hline 161 & 650600 & 230250 & 21.8 & 1,011 & 1,500 & 218 & 68 & 98.42 & 8.55 & 234 & 600 & 147 & 10 & 1.48 \\
\hline
\end{tabular}

Void cells correspond to not measured. Values for $\mathrm{Na}^{+}$and $\mathrm{K}^{+}$were tabulated in the archive as sum $\left(\mathrm{Na}^{+}+\mathrm{K}^{+}\right)$, both in meq/L and $\mathrm{mg} / \mathrm{L}$. From these data, individual values for $\mathrm{Na}^{+}$and $\mathrm{K}^{+}$were recovered, and SAR values checked. Three values of SAR were found to be in error in the original archive and were corrected

$T D S$ total dissolved salts, SAR sodium adsorption ratio $\mathrm{mmol}^{1 / 2} \mathrm{~L}^{-1 / 2}$

* Coordinates Lambert Nord Algérien

The deep groundwater is normally of low salinity (FAO 1970). The high values registered in 1996 can be ascribed either to the small depth of some boreholes that do not reach the deep groundwater table or to purely technical problems during the realization of the borehole and bad cementation between the tube and the shallow aquifer.

\section{Contamination of groundwater by nitrates}

In this region, groundwater is the only resource for human and animal drinking and for irrigation. From Table 4, it appears that average values for $\mathrm{NO}_{3}^{-}$are not significantly different. To extract information from data, the distributions of results must be studied in more detail.

Archive data from wells (FAO 1973) (Table 4) show that $50 \%$ of samples was already over $50 \mathrm{mg}_{\mathrm{NO}_{3}^{-}} \mathrm{L}^{-1}$ in 1967 , and $25 \%$ was over $100 \mathrm{mg}_{\mathrm{NO}_{3}^{-}} \mathrm{L}^{-1}$; conversely $21 \%$ was under $20 \mathrm{mg}_{\mathrm{NO}_{3}^{-}} \mathrm{L}^{-1}$, and indeed $12 \%$ of samples was analyzed and showed null values. Rainwater contains nonnull values of nitrate in the range $1 \mathrm{mg}_{\mathrm{NO}_{3}^{-}} \mathrm{L}^{-1}$ to $2 \mathrm{mg}_{\mathrm{NO}_{3}^{-}} \mathrm{L}^{-1}$ (Barbier 2011), due to natural oxidation of atmospheric N. This implies that in some cases, biogeochemical cycles are efficient enough to absorb all nitrates present in soil. There existed thus in 1967 both small values, less than $c a .20 \mathrm{mg}_{\mathrm{NO}_{3}^{-}} \mathrm{L}^{-1}$, and very large values.

The map of nitrate concentration in wells in 1967 (Fig. 3) shows the concentration increases from the exterior to the centre of the area, where rural population is concentrated. Large values are observed in the south and in the east where populations are gathered near urban areas. This can thus be ascribed to contamination. This contamination may be both from human and animal origin, as a large part of cattle was brought back to the village every night, agriculture being non-existent in 1967.

The data from boreholes in 1996 show $12 \%$ of samples over $100 \mathrm{mg}_{\mathrm{NO}_{3}^{-}} \mathrm{L}^{-1}, 24 \%$ of samples over $50 \mathrm{mg}_{\mathrm{NO}_{3}^{-}} \mathrm{L}^{-1}$, and $8 \%$ under $20 \mathrm{mg}_{\mathrm{NO}_{3}^{-}} \mathrm{L}^{-1}$. Comparison of the cumulative frequencies of nitrate concentrations (Fig. 6) clearly shows a shift towards larger values from 1996 to 2008 . Though detailed knowledge of the exact nature of aquifer exploited by every borehole is lacking, large nitrate concentrations are observed even at depths greater than $50 \mathrm{~m}$ (Fig. 7), which shows the qualitative degradation of the deep aquifer. This can be ascribed to the development of irrigated crops in the 1970s (Sebhi 1987; Mimoune 1995), to the increase of population and land use change. Large values are indeed observed in two situations: (1) in the west 
Table 2 Chemical composition of groundwater from boreholes, 1996 (ANRH 1996)

\begin{tabular}{|c|c|c|c|c|c|c|c|c|c|c|c|c|c|c|}
\hline \multirow[t]{2}{*}{ Site no. } & \multicolumn{2}{|c|}{ Coordinates* } & \multirow[t]{2}{*}{$\mathrm{pH}$} & \multirow[t]{2}{*}{$\mathrm{EC}\left(\mu \mathrm{S} \mathrm{cm}{ }^{-1}\right)$} & \multirow[t]{2}{*}{$\operatorname{TDS}\left(\mathrm{mg} \mathrm{L}^{-1}\right)$} & \multirow[t]{2}{*}{$\mathrm{Ca}^{2+}$} & \multirow[t]{2}{*}{$\mathrm{Mg}^{2+}$} & \multirow[t]{2}{*}{$\mathrm{Na}^{+}$} & \multirow[t]{2}{*}{$\mathrm{K}^{+}$} & \multirow[t]{2}{*}{$\mathrm{HCO}_{3}^{-}$} & \multirow[t]{2}{*}{$\mathrm{SO}_{4}^{2-}$} & \multirow[t]{2}{*}{$\mathrm{Cl}^{-}$} & \multirow[t]{2}{*}{$\mathrm{NO}_{3}^{-}$} & \multirow[t]{2}{*}{ SAR } \\
\hline & $X$ & $Y$ & & & & & & & & & & & & \\
\hline 907 & 641775 & 220750 & 7.4 & 2,300 & 1,603 & 88 & 90 & 233 & 7 & 88 & 588 & 343 & 22 & 4.15 \\
\hline 916 & 642350 & 223250 & 7.3 & 2,000 & 1,575 & 128 & 96 & 143 & 6 & 95 & 378 & 388 & 48 & 2.32 \\
\hline 947 & 644800 & 232300 & 7.2 & 2,000 & 1,639 & 163 & 88 & 133 & 6 & 145 & 563 & 240 & 47 & 2.08 \\
\hline 942 & 644250 & 231700 & 7 & 1,800 & 1,416 & 141 & 96 & 83 & 5 & 172 & 600 & 115 & 38 & 1.32 \\
\hline 943 & 644500 & 232700 & 7.1 & 1,500 & 1,066 & 192 & 69 & 78 & 4 & 94 & 488 & 125 & 44 & 1.22 \\
\hline 949 & 645100 & 231800 & 8 & 1,600 & 1,285 & 180 & 68 & 73 & 4 & 291 & 490 & 125 & 48 & 1.17 \\
\hline 956 & 645350 & 230775 & 7 & 3,200 & 2,091 & 257 & 114 & 178 & 8 & 66 & 988 & 333 & 42 & 2.32 \\
\hline 957 & 645325 & 230850 & 7.2 & 4,800 & 3,886 & 332 & 234 & 420 & 4 & 149 & 1,815 & 625 & 11 & 4.30 \\
\hline 959 & 647250 & 231075 & 7.7 & 2,100 & 1,408 & 220 & 66 & 73 & 3 & 198 & 608 & 145 & 49 & 1.11 \\
\hline 972 & 647800 & 231450 & 7.3 & 4,500 & 4,160 & 440 & 241 & 395 & 18 & 71 & 1,925 & 650 & 12 & 3.74 \\
\hline 974 & 646700 & 231225 & 7.2 & 7,100 & 5,730 & 332 & 302 & 750 & 23 & 108 & 2,350 & 1,025 & 12 & 7.14 \\
\hline 982 & 647400 & 231550 & 6.9 & 1,900 & 1,337 & 158 & 81 & 75 & 5 & 51 & 638 & 125 & 43 & 1.20 \\
\hline 991 & 646350 & 233100 & 7 & 1,800 & 1,273 & 172 & 79 & 65 & 7 & 203 & 563 & 100 & 44 & 1.03 \\
\hline 1000 & 646250 & 232600 & 7 & 1,600 & 1,284 & 140 & 67 & 75 & 7 & 82 & 563 & 98 & 46 & 1.30 \\
\hline 1005 & 646900 & 232900 & 7.3 & 1,700 & 1,275 & 164 & 72 & 70 & 3 & 106 & 525 & 155 & 39 & 1.14 \\
\hline 1009 & 645100 & 233100 & 6.9 & 1,600 & 1,075 & 127 & 69 & 68 & 7 & 68 & 558 & 108 & 43 & 1.20 \\
\hline 1019 & 644700 & 233300 & 7.2 & 1,700 & 1,197 & 141 & 77 & 70 & 5 & 144 & 495 & 115 & 44 & 1.17 \\
\hline 1031 & 646400 & 234925 & 6.9 & 1,600 & 1,177 & 133 & 75 & 100 & 6 & 102 & 550 & 140 & 38 & 1.71 \\
\hline 1035 & 648150 & 229250 & 7 & 3,900 & 2,710 & 229 & 144 & 318 & 10 & 79 & 1,188 & 458 & 37 & 4.04 \\
\hline 1042 & 648950 & 229450 & 7 & 2,300 & 1,503 & 293 & 79 & 108 & 5 & 65 & 695 & 213 & 39 & 1.44 \\
\hline 1046 & 648700 & 230350 & 7 & 3,100 & 2,361 & 231 & 118 & 215 & 9 & 78 & 983 & 350 & 26 & 2.86 \\
\hline 1051 & 647750 & 228200 & 7 & 2,300 & 1,476 & 227 & 80 & 93 & 5 & 120 & 670 & 230 & 48 & 1.35 \\
\hline 1054 & 647500 & 228000 & 7 & 1,800 & 1,269 & 190 & 71 & 73 & 1 & 78 & 295 & 155 & 40 & 1.14 \\
\hline 1058 & 647150 & 227800 & 7.3 & 6,900 & 5,080 & 754 & 265 & 328 & 7 & 160 & 2,350 & 865 & 130 & 2.61 \\
\hline 1066 & 646500 & 226400 & 7.2 & 2,500 & 1,943 & 238 & 113 & 120 & 6 & 138 & 900 & 200 & 25 & 1.60 \\
\hline 1070 & 645850 & 227050 & 7 & 3,000 & 1,998 & 288 & 113 & 133 & 4 & 79 & 745 & 475 & 36 & 1.68 \\
\hline 1081 & 646450 & 227750 & 7 & 4,500 & 3,268 & 401 & 179 & 250 & 3 & 60 & 1,415 & 550 & 39 & 2.60 \\
\hline 1091 & 646600 & 229000 & 7.1 & 1,500 & 1,153 & 153 & 61 & 68 & 3 & 71 & 463 & 160 & 39 & 1.17 \\
\hline 1094 & 646075 & 228700 & 7 & 1,900 & 1,379 & 183 & 71 & 65 & 4 & 205 & 525 & 145 & 51 & 1.03 \\
\hline 1104 & 644800 & 227950 & 6.9 & 3,300 & 2,873 & 332 & 151 & 208 & 5 & 92 & 1,138 & 450 & 36 & 2.37 \\
\hline 1109 & 643150 & 224450 & 7.1 & 2,800 & 2,335 & 290 & 126 & 123 & 5 & 90 & 813 & 385 & 240 & 1.51 \\
\hline 1118 & 642350 & 230350 & 7 & 2,200 & 1,766 & 188 & 112 & 135 & 4 & 85 & 808 & 213 & 45 & 1.92 \\
\hline 1125 & 643650 & 228175 & 7.6 & 2,100 & 1,379 & 207 & 84 & 78 & 3 & 138 & 625 & 193 & 59 & 1.15 \\
\hline 1127 & 673950 & 288350 & 7.2 & 1,800 & 1,243 & 160 & 76 & 73 & 1 & 162 & 482 & 150 & 80 & 1.19 \\
\hline 1134 & 640675 & 231600 & 7.1 & 7,100 & 6,131 & 584 & 465 & 475 & 17 & 196 & 2,900 & 800 & 310 & 3.54 \\
\hline 1145 & 639700 & 231500 & 7 & 4,600 & 3,635 & 431 & 244 & 220 & 12 & 113 & 1,315 & 790 & 22 & 2.09 \\
\hline 1159 & 638800 & 231300 & 7.2 & 3,200 & 3,295 & 334 & 213 & 250 & 10 & 163 & 1,225 & 600 & 22 & 2.62 \\
\hline 1168 & 638950 & 232650 & 7.3 & 3,600 & 2,725 & 301 & 169 & 195 & 10 & 268 & 975 & 450 & 34 & 2.22 \\
\hline 1171 & 637650 & 231200 & 7.3 & 3,900 & 4,006 & 308 & 218 & 238 & 12 & 110 & 1,575 & 365 & 38 & 2.53 \\
\hline 1183 & 637200 & 231175 & 7 & 4,200 & 2,915 & 280 & 214 & 225 & 17 & 88 & 1,250 & 525 & 23 & 2.45 \\
\hline 1190 & 637550 & 232050 & 7 & 9,700 & 6,608 & 758 & 431 & 615 & 7 & 69 & 2,430 & 1,850 & 78 & 4.40 \\
\hline 1191 & 637750 & 232450 & 7 & 4,100 & 2,835 & 241 & 214 & 245 & 17 & 87 & 1,115 & 575 & 18 & 2.76 \\
\hline 1205 & 637100 & 238650 & 7.9 & 3,500 & 2,161 & 104 & 85 & 465 & 16 & 426 & 583 & 513 & 25 & 8.16 \\
\hline 1254 & 640150 & 228800 & 7 & 1,800 & 1,206 & 223 & 54 & 33 & 2 & 88 & 675 & 75 & 54 & 0.51 \\
\hline 1257 & 639925 & 228450 & 6.8 & 2,300 & 2,099 & 334 & 299 & 38 & 4 & 95 & 895 & 125 & 195 & 0.36 \\
\hline 1267 & 638250 & 228450 & 7.3 & 3,500 & 1,801 & 225 & 105 & 118 & 4 & 88 & 783 & 230 & 185 & 1.62 \\
\hline 1276 & 637350 & 227800 & 7.1 & 3,400 & 2,460 & 295 & 152 & 170 & 13 & 109 & 1,113 & 308 & 125 & 2.00 \\
\hline
\end{tabular}


Table 2 continued

\begin{tabular}{|c|c|c|c|c|c|c|c|c|c|c|c|c|c|c|}
\hline \multirow[t]{2}{*}{ Site no. } & \multicolumn{2}{|c|}{ Coordinates* } & \multirow[t]{2}{*}{$\mathrm{pH}$} & \multirow[t]{2}{*}{$\mathrm{EC}\left(\mu \mathrm{S} \mathrm{cm}{ }^{-1}\right)$} & \multirow[t]{2}{*}{$\operatorname{TDS}\left(\mathrm{mg} \mathrm{L}^{-1}\right)$} & \multirow[t]{2}{*}{$\mathrm{Ca}^{2+}$} & \multirow[t]{2}{*}{$\mathrm{Mg}^{2+}$} & \multirow[t]{2}{*}{$\mathrm{Na}^{+}$} & \multirow[t]{2}{*}{$\mathrm{K}^{+}$} & \multirow[t]{2}{*}{$\mathrm{HCO}_{3}^{-}$} & \multirow[t]{2}{*}{$\mathrm{SO}_{4}^{2-}$} & \multirow[t]{2}{*}{$\mathrm{Cl}^{-}$} & \multirow[t]{2}{*}{$\mathrm{NO}_{3}^{-}$} & \multirow[t]{2}{*}{ SAR } \\
\hline & $X$ & $Y$ & & & & & & & & & & & & \\
\hline 1279 & 636800 & 229200 & 7 & 3,600 & 2,715 & 276 & 187 & 238 & 15 & 126 & 1,088 & 488 & 30 & 2.70 \\
\hline 1291 & 637600 & 228950 & 7 & 2,800 & 2,029 & 217 & 110 & 160 & 10 & 123 & 800 & 285 & 72 & 2.20 \\
\hline 1304 & 634450 & 230550 & 7.6 & 1,800 & 1,344 & 94 & 101 & 140 & 10 & 204 & 488 & 218 & 30 & 2.38 \\
\hline
\end{tabular}

$T D S$ total dissolved salts, $S A R$ sodium adsorption ratio $\mathrm{mmol}^{1 / 2} \mathrm{~L}^{-1 / 2}$

* Coordinates Lambert Nord Algérien

Table 3 Chemical composition of groundwater from boreholes, 2008 (This study)

\begin{tabular}{|c|c|c|c|c|c|c|c|c|c|c|c|c|c|c|c|c|}
\hline \multirow{2}{*}{$\begin{array}{l}\text { Site } \\
\text { no. }\end{array}$} & \multicolumn{2}{|c|}{ Coordinates* } & \multirow{2}{*}{$\begin{array}{l}\text { Elev. } \\
(\mathrm{m})\end{array}$} & \multirow[t]{2}{*}{$\mathrm{pH}$} & \multirow[t]{2}{*}{$t\left({ }^{\circ} \mathrm{C}\right)$} & \multirow[t]{2}{*}{ EC } & \multirow{2}{*}{$\begin{array}{l}\text { TDS } \\
\left(\mathrm{mg} \mathrm{L}^{-1}\right)\end{array}$} & \multirow[t]{2}{*}{$\mathrm{Ca}^{2+}$} & \multirow[t]{2}{*}{$\mathrm{Mg}^{2+}$} & \multirow[t]{2}{*}{$\mathrm{Na}^{+}$} & \multirow[t]{2}{*}{$\mathrm{K}^{+}$} & \multirow[t]{2}{*}{$\mathrm{HCO}_{3}^{-}$} & \multirow[t]{2}{*}{$\mathrm{SO}_{4}^{2-}$} & \multirow[t]{2}{*}{$\mathrm{Cl}^{-}$} & \multirow[t]{2}{*}{$\mathrm{NO}_{3}^{-}$} & \multirow[t]{2}{*}{ SAR } \\
\hline & $X$ & Y & & & & & & & & & & & & & & \\
\hline 1 & 655472.99 & 221217.15 & 454 & 7.40 & 21 & 803 & 666 & 92.82 & 30.89 & 37.04 & 0.68 & 158.6 & 78 & 95 & 118 & 0.85 \\
\hline 2 & 655165.07 & 221660.96 & 449 & 7.47 & 20.9 & 844 & 786 & 112.48 & 33.36 & 28.25 & 0.66 & 158.6 & 102 & 125 & 134 & 0.60 \\
\hline 3 & 655313.14 & 222294.05 & 444 & 7.44 & 21.3 & 644 & 512 & 88.93 & 26.89 & 23.86 & 0.46 & 152.6 & 74 & 70 & 106 & 0.57 \\
\hline 4 & 654848.15 & 222790.60 & 448 & 7.40 & 21.1 & 950 & 870 & 112.93 & 42.54 & 63.87 & 0.37 & 189.1 & 210 & 125 & 82 & 1.30 \\
\hline 5 & 654518.40 & 222664.69 & 451 & 7.31 & 22 & 1,688 & 1,272 & 216.95 & 67.74 & 62.39 & 1.03 & 183 & 230 & 330 & 150 & 0.94 \\
\hline 6 & 653272.82 & 222635.66 & 455 & 7.28 & 21.9 & 1,369 & 1,358 & 200.5 & 60.47 & 93.37 & 0.82 & 183 & 500 & 155 & 92 & 1.48 \\
\hline 7 & 651665.76 & 222646.86 & 454 & 7.17 & 21.5 & 2,500 & 2,510 & 280.41 & 101.26 & 140.14 & 0.64 & 152.5 & 760 & 295 & 6 & 1.82 \\
\hline 8 & 656815.70 & 224341.16 & 445 & 7.32 & 21 & 1,893 & 1,510 & 190.98 & 91.12 & 141.37 & 1.59 & 225.7 & 476 & 225 & 158 & 2.10 \\
\hline 9 & 654693.46 & 226446.56 & 417 & 7.21 & 22 & 2,520 & 1,680 & 210.7 & 100.89 & 191.19 & 1 & 195.2 & 548 & 360 & 60 & 2.70 \\
\hline 20 & 660271.67 & 226843.36 & 432 & 7.27 & 21 & 1,573 & 1,208 & 180.34 & 60.46 & 116.84 & 1.06 & 183 & 500 & 160 & 42 & 1.92 \\
\hline 21 & 660373.88 & 223838.65 & 445 & 7.10 & 22.1 & 2,780 & 2,250 & 321.3 & 118.58 & 232.18 & 1.28 & 207.4 & 796 & 405 & 88 & 2.80 \\
\hline 22 & 660307.98 & 222066.75 & 445 & 7.30 & 22.4 & 909 & & & 43.2 & 72.24 & 0.44 & 231.8 & 242 & 90 & 36 & 1.49 \\
\hline 30 & 656817.26 & 227678.25 & 416 & 7.37 & 20.7 & 1,366 & 1,196 & 142.45 & 48.54 & 130.18 & 0.73 & 225.7 & 404 & 130 & 44 & 2.40 \\
\hline 31 & 657026.41 & 228016.50 & 414 & 7.33 & 21.2 & 1,510 & 1,242 & 161.28 & 66.51 & 122.36 & 0.78 & 219.6 & 500 & 175 & 44 & 2.04 \\
\hline 32 & 657909.45 & 228712.55 & 407 & 7.26 & 22.4 & 1,426 & 1,196 & 173.36 & 62.08 & 112.36 & 1.03 & 244 & 500 & 115 & 36 & 1.86 \\
\hline 33 & 651419.74 & 224092.11 & 451 & 7.20 & 21.8 & 2,430 & 1,746 & 264.57 & 91.86 & 177.86 & 1.93 & 183 & 744 & 260 & 66 & 2.39 \\
\hline 34 & 650740.94 & 226400.69 & 444 & 7.20 & 22.1 & 2,540 & 1,492 & 249.6 & 75.64 & 126.5 & 0.51 & 164.7 & 620 & 200 & 76 & 1.79 \\
\hline 41 & 655283.71 & 228649.51 & 412 & 7.22 & 24.5 & 1,643 & 1,326 & 215.4 & 57.81 & 58.88 & 0.55 & 207.4 & 450 & 155 & 35 & 0.92 \\
\hline
\end{tabular}

$T D S$ total dissolved salts, SAR sodium adsorption ratio

* Coordinates Lambert Nord Algérien; Elev. = elevation above sea level; EC: $/ \mu \mathrm{S} \mathrm{cm}{ }^{-1}$

(Maârif, Fig. 4) where there exists an agglomeration, close to the limit of commons, but not an agricultural area (compare with Fig. 1, white area); (2) in agricultural areas north and south of Maârif (green areas in Fig. 1).

It is worthwhile to note the spatial coincidence of the contaminated areas of both periods, which suggests strongly a communication between the two aquifers.

Nitrate concentration ranges from $6 \mathrm{mg}_{\mathrm{NO}_{3}^{-}} \mathrm{L}^{-1}$ to $158 \mathrm{mg}_{\mathrm{NO}_{3}^{-}} \mathrm{L}^{-1}$, in 2008 , with $27 \%$ over $100 \mathrm{mg}_{\mathrm{NO}_{3}^{-}} \mathrm{L}^{-1}$, $61 \%$ of samples over $50 \mathrm{mg}_{\mathrm{NO}_{3}^{-}} \mathrm{L}^{-1}$, only 1 sample $(5 \%)$ under $20 \mathrm{mg}_{\mathrm{NO}_{3}^{-}} \mathrm{L}^{-1}$. Though the direct comparison of average values showed no significant differences, the detailed analysis of the distribution shows an extension of contamination of groundwater by nitrate. This extension progresses both vertically and laterally. Evidence for this is the fact that small values (under $20 \mathrm{mg}_{\mathrm{NO}_{3}^{-}} \mathrm{L}^{-1}$ ) were observed in the deep aquifer in 1996 and are no more observed in 2008. This indicates a contamination seriously constraining agricultural development and public health. This contamination is of anthropic origin, and could be due to fertilizer input and cattle breeding, or to seepage of water from septic tanks. This hazard is enhanced by sandy texture and direct gravitary irrigation, as irrigation is always in excess as compared to plant requirements and water holding capacity.

The following measures can be taken as a first step in the present irrigation and crop system to remediate to the contamination: (1) water control should avoid exceeding water holding capacity, while considering the necessity of a salt leaching fraction; (2) N-fertilizers should be fractionated and based upon the plant requirements and soil 
Table 4 Synthetic statistical parameters for the chemical composition of groundwaters of Hodna from 1967 to present (2008)

\begin{tabular}{|c|c|c|c|c|c|}
\hline Parameter & Mean & Median & Minimum & Maximum & Standard deviation \\
\hline \multicolumn{6}{|c|}{$1967[N=63$, except for $\mathrm{t}(N=59)$, nitrate $(N=56)$ and EC $(N=62)]$} \\
\hline$t\left({ }^{\circ} \mathrm{C}\right)$ & 17.55 & 18.00 & 12.8 & 21.8 & 2.13 \\
\hline $\mathrm{Ca}^{2+}\left(\mathrm{mg} \mathrm{L}^{-1}\right)$ & 411.05 & 416 & 30 & 898 & 208.77 \\
\hline $\mathrm{Mg}^{2+}$ & 277.41 & 219 & 48 & 1,340 & 226.55 \\
\hline $\mathrm{Na}^{+}$ & 520 & 300 & 24 & 3,456 & 600 \\
\hline $\mathrm{K}^{+}$ & 25 & 9 & 0 & 99 & 29 \\
\hline $\mathrm{HCO}_{3}^{-}$ & 204.62 & 206 & 38 & 424 & 74.89 \\
\hline $\mathrm{SO}_{4}^{2-}$ & $1,882.03$ & 1,810 & 185 & 7,488 & $1,378.04$ \\
\hline $\mathrm{Cl}^{-}$ & 733.43 & 426 & 57 & 4,473 & 837.41 \\
\hline $\mathrm{NO}_{3}^{-}$ & 71.41 & 51 & 0 & 535 & 79.32 \\
\hline $\mathrm{CE}\left(\mu \mathrm{S} \mathrm{cm}{ }^{-1}\right)$ & $5,036.55$ & 4,025 & 1,011 & 21,900 & $3,793.65$ \\
\hline $\operatorname{TDS}\left(\mathrm{mg} \mathrm{L}^{-1}\right)$ & 4,572 & 3,924 & 738 & 20,300 & $3,530.22$ \\
\hline SAR & 3.76 & 2.7 & 0.33 & 18.03 & 3.26 \\
\hline \multicolumn{6}{|l|}{$1996(N=50)$} \\
\hline $\mathrm{Ca}^{2+}\left(\mathrm{mg} \mathrm{L}^{-1}\right)$ & 257.54 & 226 & 88 & 758 & 141.9 \\
\hline $\mathrm{Mg}^{2+}$ & 141.76 & 107 & 54 & 465 & 92.31 \\
\hline $\mathrm{Na}^{+}$ & 185.9 & 134 & 33 & 750 & 150.52 \\
\hline $\mathrm{K}^{+}$ & 7.58 & 6 & 1 & 23 & 4.99 \\
\hline $\mathrm{HCO}_{3}^{-}$ & 125.72 & 104 & 51 & 426 & 68.59 \\
\hline $\mathrm{SO}_{4}^{2-}$ & 959.8 & 764 & 295 & 2,900 & 587.75 \\
\hline $\mathrm{Cl}^{-}$ & 366.88 & 262.5 & 75 & 1,850 & 313.01 \\
\hline $\mathrm{NO}_{3}^{-}$ & 58.02 & 41 & 11 & 310 & 58.44 \\
\hline $\mathrm{CE}\left(\mu \mathrm{S} \mathrm{cm}{ }^{-1}\right)$ & 3,076 & 2,400 & 1,500 & 9,700 & $1,707.67$ \\
\hline $\operatorname{TDS}\left(\mathrm{mg} \mathrm{L}^{-1}\right)$ & $2,323.56$ & 1,872 & 1,066 & 6,608 & $1,355.33$ \\
\hline $\mathrm{pH}$ & 7.15 & 7.05 & 6.8 & 8 & 0.25 \\
\hline SAR & 2.24 & 1.96 & 0.36 & 8.16 & 1.48 \\
\hline \multicolumn{6}{|l|}{$2008(N=18)$} \\
\hline$t\left({ }^{\circ} \mathrm{C}\right)$ & 21.72 & 21.65 & 20.7 & 24.5 & 0.88 \\
\hline $\mathrm{Ca}^{2+}\left(\mathrm{mg} \mathrm{L}^{-1}\right)$ & 184.43 & 185.66 & 88.93 & 321.3 & 67.83 \\
\hline $\mathrm{Mg}^{2+}$ & 65.55 & 61.28 & 26.89 & 118.58 & 26.53 \\
\hline $\mathrm{Na}^{+}$ & 107.27 & 114.6 & 23.86 & 232.18 & 57.86 \\
\hline $\mathrm{K}^{+}$ & 0.86 & 0.76 & 0.37 & 1.93 & 0.41 \\
\hline $\mathrm{HCO}_{3}^{-}$ & 192.49 & 186.05 & 152.5 & 244 & 28.92 \\
\hline $\mathrm{SO}_{4}^{2-}$ & 429.67 & 488 & 74 & 796 & 229.61 \\
\hline $\mathrm{Cl}^{-}$ & 192.78 & 157.5 & 70 & 405 & 98.97 \\
\hline $\mathrm{NO}_{3}^{-}$ & 76.28 & 71 & 6 & 158 & 43.39 \\
\hline $\mathrm{CE}\left(\mu \mathrm{S} \mathrm{cm}{ }^{-1}\right)$ & $1,632.67$ & 1541.5 & 644 & 2,780 & 680.2 \\
\hline $\operatorname{TDS}\left(\mathrm{mg} \mathrm{L}^{-1}\right)$ & $1,313.56$ & 1,257 & 512 & 2,510 & 515.89 \\
\hline $\mathrm{pH}$ & 7.29 & 7.29 & 7.1 & 7.47 & 0.1 \\
\hline SAR & 1.67 & 1.81 & 0.57 & 2.8 & 0.69 \\
\hline
\end{tabular}

1967 phreatic groundwater, 1996 and 2008 deep groundwater

analyses; (3) soil surface should be covered during the whole year.

As a second step, new irrigation techniques, more efficient such as localized irrigation should be implemented to minimize water requirements and preserve water quality. This implies training farmers, who are numerous in the Southern Hodna region (about 5,000), with mid-term and long term programs. Further research should be initiated on N-cycle and $\mathrm{N}$-fertilization, taking into account the specificities of arid conditions, to obtain local agronomic references. 


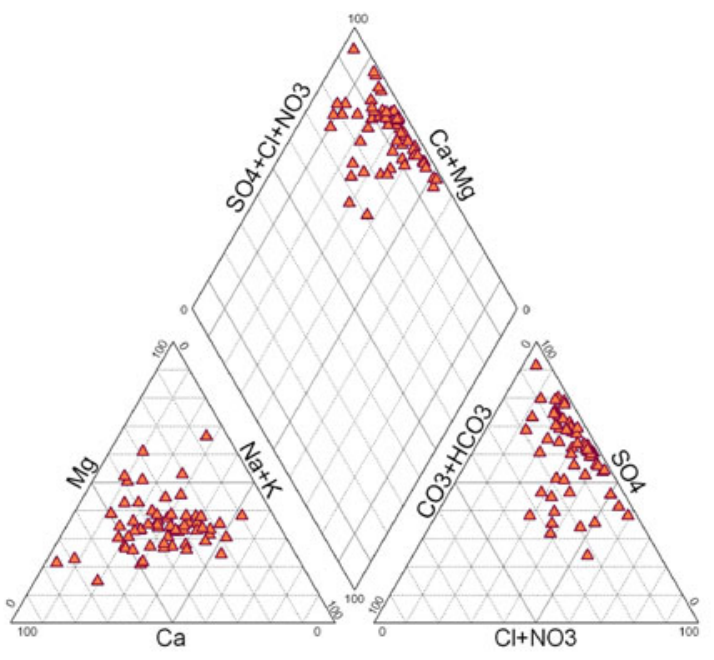

(a) Wells, 1967.

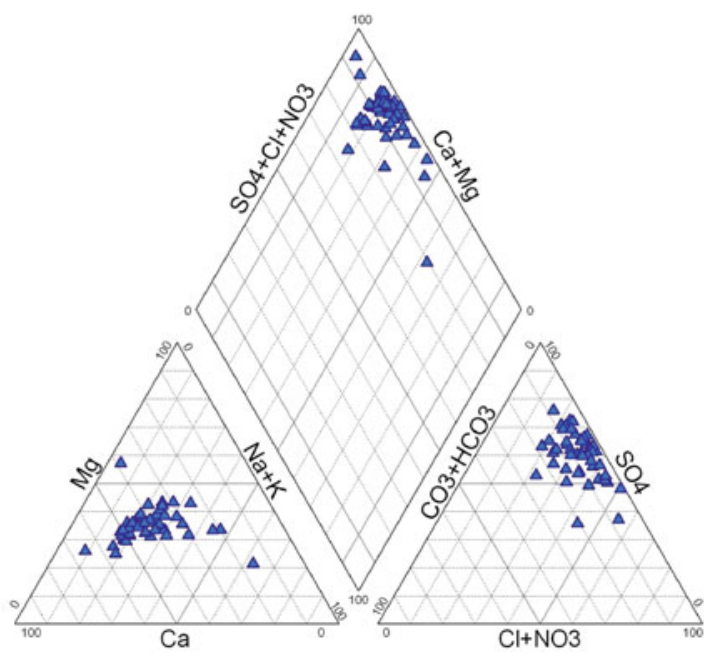

(b) Boreholes, 1996.

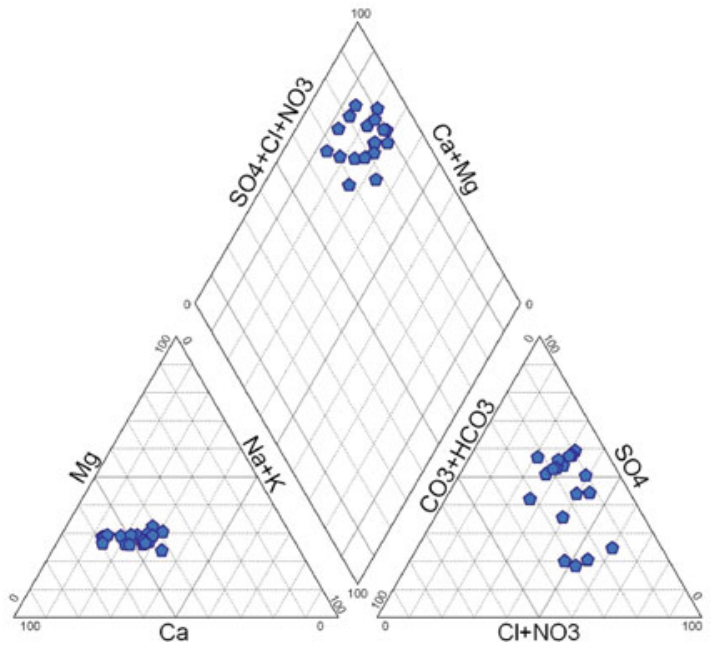

(c) Boreholes, 2008 .

Fig. 5 Piper's diagrams for groundwaters from Chott El Hodna

Fig. 6 Distribution of nitrate concentrations in 1996 and 2008. Cumulative frequency versus nitrate concentration

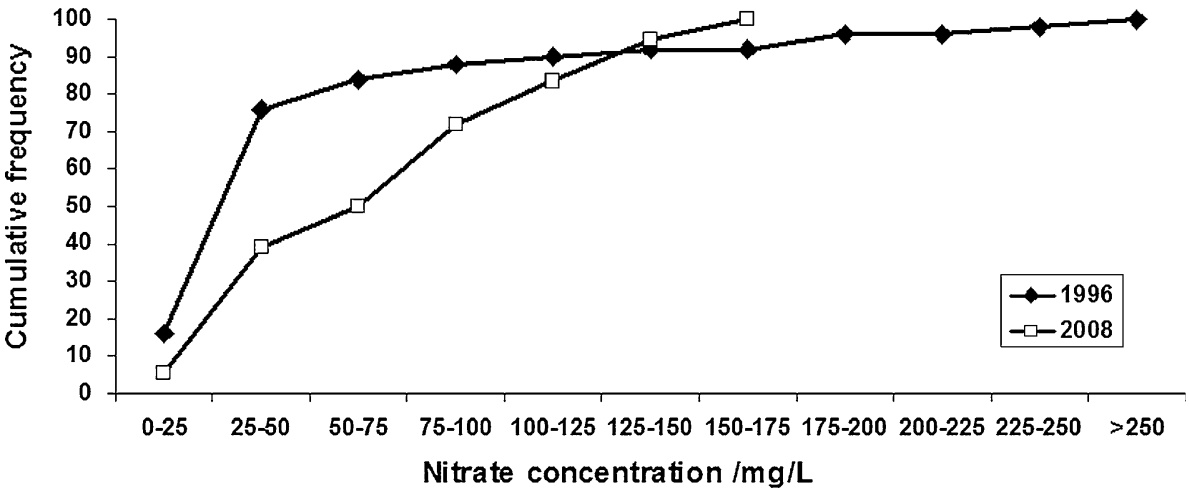

\section{Conclusions}

The Hodna region, an arid zone dedicated to pastoralism under severe climatic conditions, has been intensively anthropized as a consequence of agricultural policies of the past decades. Arable land areas increased at the expense of grazing lands on the basis of exploitation of water resources. Salinity of borehole water is moderate, with high 
Fig. 7 Nitrate concentration in boreholes of increasing depth. Data from ANRH (1996)

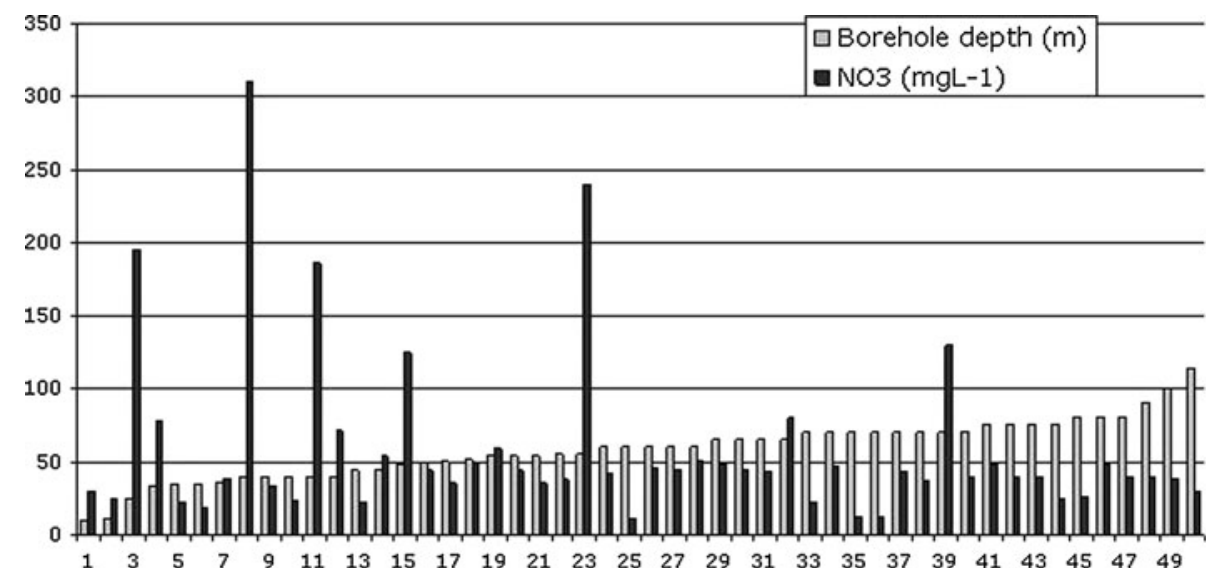

values in the west of the agricultural area, where $\mathrm{Cl}^{-}$ concentrations override regulation thresholds. Waters from recent boreholes are less concentrated. Sodisation hazard is negligible, as estimated by SAR.

However, nitrate contamination affected about half of samples of the phreatic aquifer as early as 1967 (wells), while pastoralism was then dominant. Intensification of agriculture resulted in an increase of nitrate contamination of the deep aquifer, which is the only water resource for irrigation, human and animal needs. This could impair the sustainability of the development of the region.

To remediate this degradation of water quality, measures are proposed at two different time scales. First, in the present irrigation and cropping system, to fractionate $\mathrm{N}$ inputs and better control water inputs. Secondly, implement more efficient and less resource consuming techniques, such as localized irrigation, with adaptation of crop systems and fertilizer inputs to arid conditions, based upon farmers' training programs and local agronomic references, that are presently lacking.

Acknowledgments The Agence Nationale des Ressources Hydrauliques (ANRH) and the Haut Commissariat au Développement de la Steppe de Djelfa (HCDS) are gratefully acknowledged for communication of archive data. An anonymous reviewer and Dr W. LaMoreaux are gratefully acknowledged too for their comments that helped improve the manuscript.

Open Access This article is distributed under the terms of the Creative Commons Attribution License which permits any use, distribution, and reproduction in any medium, provided the original author(s) and the source are credited.

\section{References}

Abdesselam S, Merabet Y, Halitim A (2007) Vulnerability of the functions of an agro-pastoral ecosystem to climate change (Case of Southern Hodna) (in French). In: Journées internationales "L'impact des changements climatiques sur les régions arides et semi-arides", CRSTRA, Biskra, Algérie
Abdesselam S, Merabet Y, Halitim A (2008) Cartography of vulnerability to desertification in Southern Hodna (in French). In: Situation et valorisation de la steppe en Algérie, Université de Tiaret, Algérie

Abdesselam S, Amor Z, Halouan R (2009) Dynamics of land use in the arid zone-Case of Southern Hodna (in French). In: La protection et préservation des écosystèmes sahariens, Université de Ouargla, Algérie

Al Droubi A, Fritz B, Gac JY, Tardy Y (1980) Generalized residual alkalinity concept; application to prediction of the chemical evolution of natural waters by evaporation. Am J Sci 280:560-572

ANRH (1996) Survey of water wells and boreholes and of flows of the Chott El-Hodna (in French). Tech. rep., Agence Nationale des Ressources Hydrauliques, Algérie

ANRH (2006) Modelling of the Hodna aquifer. Mission I: data collection, analysis and synthesis (in French). Tech. rep., Agence Nationale des Ressources Hydrauliques, Algérie, Icosium forage et engineering services

ANRH (2007) Modelling of the Hodna aquifer. Mission III model exploitation. Sub-Mission III 2 predictive simulation and proposal for optimum management of water resources (in French). Tech. rep., Agence Nationale des Ressources Hydrauliques, Icosium forage et engineering services

Ayers RS, Westcot DW (1985) Water quality for agriculture. FAO Irrigation and Drainage Paper 29 (Rev. 1)

Baali F, Rouabhia A, Kherici N, Djabri L, Bouchaou L, Hani A (2007) Underground water quality and contamination hazards in semi-arid conditions. Case of the Chéria basin, NE Algeria (in French). Estudios Geológicos 63:127-133

Barbier J (2011) Tell me, little spring... some secrets of Naiads (in French). Les Éditions Persée, New York

Boyadgiev TG (1975) Soils of Hodna (in French). Tech. Rep. Rapport Algérie 9, FAO, Rome

Breemen NV, Wielemaker W (1974) Buffer intensities and equilibrium $\mathrm{pH}$ of minerals and soils. Soil Sci Soc Am Proc 38:55-66

Daoud Y, Halitim A (1994) Irrigation and salinity in algerian Sahara (in French). Sécheresse 5:151-160

Djili K, Daoud Y, Gaouar A, Beldjoudi Z (2003) Secondary soil salinization in Sahara: impact on agriculture sustainability in new areas of development (in French). Sécheresse 14:241-246

Durand JH (1958) Irrigable soils (in French). Service d'Étude des Sols, Alger

FAO (1968) Geophysical studies in the Chott Hodna basin (in French). Tech. rep., FAO

FAO (1970) Natural Resources Surveys, Agricultural Experimentation and Demonstration in the Hodna Region, Central Algeria, Algeria ALG/9. Tech. rep., FAO, Roma 
FAO (1972) Project on Hodna development studies, Algeria. Final report on isotopic studies applied to the hydrogeology of the Hodna basin, Algeria ALG/9. Tech. rep., FAO, Roma

FAO (1973) Hydrogeological studies in Chott El Hodna Basin. vol. II, Annex, Algeria, ALG/9 (in French). Tech. rep., FAO, Rome

Grandin G, Joly F (2008) Pediment, genesis, dynamics and correlative formations (in French). In: Dewolf Y, Bourrié G (eds) LES FORMATIONS SUPERFICIELLES, Genèse-TypologieClassification-Paysages et environnement-Ressources et risques, Ellipses, chap 6.2.6, pp 201-216

Mallet JL (2002) Geomodeling. Applied geostatistics series. Oxford University Press, New York

Mimoune S (1995) Management of salt-affected soils and desertification in an endoreic basin, South of the Chott El Hodna, Algeria (in French). PhD thesis, Université d'Aix-Marseille I
Rouabhia A, Baali F, Kherici N, Djabri L (2004) Vulnerability of the groundwater of miocene sands of El Ma El Abiod (Algeria) plain, and contamination hazards (in French). Sécheresse $15: 347-352$

Sebhi S (1987) Mutation of rural world in Algeria-case of Hodna (in French). Éditions OPU, Alger

USSL (1954) Diagnosis and improvement of saline and alkali soils. Handbook 60, U. S. Salinity Laboratory Staff, U.S. Department of Agriculture

WRB (2006) World reference base for soil resources-a framework for international classification, correlation and communication. Word soil resources reports 103, FAO, Roma 